\title{
Management of Type 2 Late Sleeve Leak by Laparoscopic Suturing and Conversion to Roux-en-Y Gastric Bypass: Video Report
}

\author{
Palanivelu Praveenraj ${ }^{1}$ • Rachel M. Gomes ${ }^{1}$ - Saravana Kumar ${ }^{1}$. \\ Palanisamy Senthilnathan ${ }^{2} \cdot$ Ramakrishnan Parthasarathi $^{2}$. \\ Subbiah Rajapandian ${ }^{2}$. Chinnusamy Palanivelu ${ }^{2}$
}

Published online: 25 July 2015

(C) Springer Science+Business Media New York 2015

\begin{abstract}
Background Laparoscopic sleeve forming gastrectomy (SFG) is a commonly performed bariatric procedure for the surgical management of morbid obesity. Staple line gastric leaks occur infrequently but are the most feared complication causing prolonged morbidity (Burgos et al., Obes Surg 19(12):16727, 2009; Márquez et al., Obes Surg 20(9):1306-11, 2010). Roux-en-Y diversion is an accepted management (Baltasar et al., Surg Obes Relat Dis 4(6):759-63, 2008). The aim of this video was to demonstrate the operative management of a late sleeve leak by laparoscopic suturing \& conversion to a RYGB. Methods We present the case of an 18-year-old woman with a BMI of $44.68 \mathrm{~kg} / \mathrm{m}^{2}$ with hypothyroidism and polycystic ovarian disease who underwent laparoscopic sleeve gastrectomy and presented with a leak on postoperative day 13. She was diagnosed to have a type 2, late leak just beyond the esophagogastric junction (Csendes et al., Hepatogastroenterology 37 Suppl 2:174-7, 1990)

Results In this multimedia high-definition video, we present stepby-step the operative management of a late sleeve leak by laparoscopic suturing and conversion to a RYGB. Procedure included mobilization of the gastric sleeve, identification and suturing of the
\end{abstract}

Electronic supplementary material The online version of this article (doi:10.1007/s11695-015-1813-5) contains supplementary material, which is available to authorized users.

Palanivelu Praveenraj

praveenraj@me.com

1 Department of Bariatric Surgery, GEM Hospital and Research Centre, 45, Pankaja Mill Road, Coimbatore 641045, India

2 Department of Surgical Gastroenterology, GEM Hospital and Research Centre, Coimbatore, India fistulous opening, creation of a gastric pouch, creation of an antecolic Roux limb, gastrojejunal anastomosis and jejuno-jejunal anastomosis. Drainage of fistula gradually decreased with absence of a leak on imaging in 12 days. This patient was diagnosed with a gastric sleeve leak on the 13th postoperative day, and the time to fistula closure from diagnosis was 1 month.

Conclusions Sleeve leak fistula repair with conversion to a RYGB aids healing by providing surgical decompression and better drainage. It may be considered as an alternative management technique in sleeve leaks.

Keywords Bariatric surgery $\cdot$ Gastric sleeve leak $\cdot$ Morbid obesity $\cdot$ Sleeve gastrectomy

Conflict of Interest The authors declare that they have no competing interests.

Statement of Informed Consent and Compliance with Ethical Standards Informed consent was obtained from all individual participants included in the study. This study has been approved by the appropriate institutional committee.

\section{References}

1. Burgos AM, Braghetto I, Csendes A, et al. Gastric leak after laparoscopic-sleeve gastrectomy for obesity. Obes Surg. 2009;19(12):1672-7.

2. Márquez MF, Ayza MF, Lozano RB, et al. Gastric leak after laparoscopic sleeve gastrectomy. Obes Surg. 2010;20(9):1306-11.

3. Baltasar A, Serra C, Bengochea M, et al. Use of Roux limb as remedial surgery for sleeve gastrectomy fistulas. Surg Obes Relat Dis. 2008;4(6):759-34.

4. Csendes A, Díaz JC, Burdiles P, et al. Classification and treatment of anastomotic leakage after extended total gastrectomy in gastric carcinoma. Hepatogastroenterology. 1990;37 Suppl 2:174-7. 